\title{
Causal Knowledge Extraction through Large-Scale Text Mining
}

\author{
Oktie Hassanzadeh, Debarun Bhattacharjya, Mark Feblowitz, \\ Kavitha Srinivas, Michael Perrone, Shirin Sohrabi, Michael Katz
}

IBM Research

\{hassanzadeh, debarunb, mfeb, mpp, ssohrab\}@us.ibm.com, \{kavitha.srinivas, michael.katz1\}@ibm.com

\begin{abstract}
In this demonstration, we present a system for mining causal knowledge from large corpuses of text documents, such as millions of news articles. Our system provides a collection of APIs for causal analysis and retrieval. These APIs enable searching for the effects of a given cause and the causes of a given effect, as well as the analysis of existence of causal relation given a pair of phrases. The analysis includes a score that indicates the likelihood of the existence of a causal relation. It also provides evidence from an input corpus supporting the existence of a causal relation between input phrases. Our system uses generic unsupervised and weakly supervised methods of causal relation extraction that do not impose semantic constraints on causes and effects. We show example use cases developed for a commercial application in enterprise risk management.
\end{abstract}

\section{Introduction}

Capturing and representing causal knowledge is a challenging problem in AI, with important applications in various domains such as healthcare, legal, and enterprise risk management. While a large body of work in AI is concerned with causal discovery and modeling (Pearl 2009; Halpern 2016), there is also a rich literature on extracting causal knowledge expressed by humans in natural language within text documents. Most prior work in this area puts restrictions on causes and effects, e.g., by restricting them to events with a particular semantic representation (Do, Chan, and Roth 2011; Radinsky, Davidovich, and Markovitch 2012). This limits the application of such approaches to cases where causes and effects can be extracted into a particular representation. Existing techniques also often require large amounts of labelled training data (Kruengkrai et al. 2017; Dasgupta et al. 2018)], which limits the application to cases where such training data can be obtained.

In this demonstration, we present a system that addresses these shortcomings, and by relying on causal statements that are prevalent in various kinds of sources (e.g., analysis reports, healthcare notes, legal and scientific literature, news articles, etc.), provides a set of tools and APIs for retrieval

Copyright (C) 2020, Association for the Advancement of Artificial Intelligence (www.aaai.org). All rights reserved. and analysis of causal knowledge. A highlight of our system is using state-of-the-art neural network based embeddings and neural language models to handle the enormous variety of representations of the same cause/effect in natural language. We have evaluated the effectiveness of using our APIs in answering binary causal questions (Hassanzadeh et al. 2019).

\section{Causal Extraction Framework}

Figure 1 shows our causal extraction framework. The input is a large corpus of text documents. The documents are ingested and turned into a large collection of sentences and phrases that are in turn used in the following two components. The ingestion and processing is performed in parallel on a distributed processing framework.

Causal Knowledge Extraction Engine This component first identifies sentences that are likely to be causal statements, and then extracts cause and effect text spans and phrases from these sentences. Each input sentence results in one or more $(X, Y)$ pairs where $X$ and $Y$ are text spans or phrases (referred to as Causal Mentions by Sharp et al. (2016)), extracted using a variety of NLP techniques. The output of this component is a collection of cause-effect pairs along with meta-data identifying the source article and sentence and the phrase extraction method (if any). This output is indexed on a distributed Information Retrieval (IR) engine that enables full-text search.

Semantic Embeddings Engine To handle the variety of representations of causes and effects in natural language, and enable effective retrieval and analysis, we use the large input corpus to build distributed representations of the words, phrases, and sentences in the corpus. For words, we use word2vec (Mikolov et al. 2013). For phrases, we use an adaptation of word2 vec based on our previous work (Hassanzadeh, Trewin, and Gliozzo 2018), by treating each sentence as a set of phrases and building embeddings that do not take the order of the phrases or the length of the sentence into account. For sentences, we use BERT (Devlin et al. 2018)-based embeddings. The vectors are then indexed using a highly efficient nearest neighbor search index. 


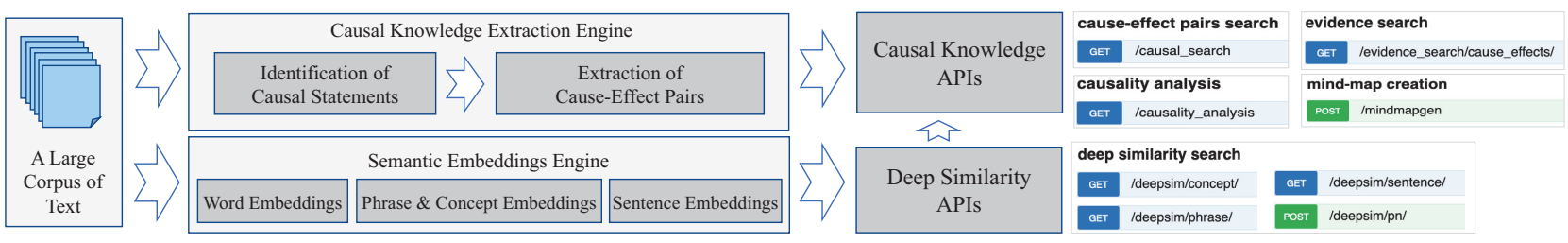

Figure 1: Causal Extraction Framework \& APIs

\section{Causal Knowledge APIs}

The key API functions implemented are as follows:

Cause-Effect Pairs Search: This API enables searching for effects of a given cause, causes of a given effect, or mentions of a given cause-effect pair. The parameters include: cause and effect (each could be '*', indicating "any"), query type (e.g., "and" or "or"), the source (e.g., News Articles Corpus), field (e.g., "title" or "body"), phrase extraction method for input cause and effect and for cause-effect pairs extraction (if any), extension method along with parameters (e.g., phrase embeddings along with model parameters).

Evidence Search: An API similar to the above with the same input parameters, but returning groups of source sentences along with meta-data (e.g., URL of the news article).

Causal Analysis: This API takes in a cause-effect pair with the same parameters as the above APIs, and returns a "Causality Score" in addition to the list of cause-effect pairs as evidences in the input corpus. The causality score is calculated in a number of different ways, specified via an additional input parameter. As an example, one method of calculating the score is by dividing the number of hits founds for $(X, Y)$ by the number of hits found for $(Y, X)$, based on the intuition that if $X$ causes $Y$, it is less likely that $Y$ causes $X$. Another method uses sentence embeddings, and returns the average similarity of the top- $k$ causal sentences to an input sentence like " $X$ may cause $Y$ " constructed from the input pair. The intuition for this score is that if $X$ causes $Y$, the constructed sentence will have a number of highly similar causal sentences in the index of causal sentences. A third approach is a combination of the first two ideas, where the average similarity score of method 2 is divided by the average similarity of the top- $k$ causal sentences to a constructed sentence like " $Y$ may cause $X$ ".

Mind Map Creation: This API creates a graph of causal knowledge given a small number of seed phrases from the target domain of interest. The API uses variations of the input phrases to query for cause-effect pairs with a high "causality score". The output of this API can be turned into a "Mind Map" for visualization. These Mind Maps are also a crucial part of the input to the IBM Scenario Planning Advisor (SPA) (Sohrabi et al. 2019), a decision support system for enterprise risk management. Creating and curating the Mind Maps manually is a labor-intensive task. Thus, automating Mind Maps creation is a valuable outcome of our application.

\section{Demonstration Plan}

For this demonstration, we will show the application of the above API functions using a number of enterprise use cases on a live deployment of our system. We use a corpus of roughly one billion sentences from public news sources as our main corpus, and demonstrate the effectiveness of our semantic embeddings and neural language model based approaches comparing with baseline solutions.

\section{References}

Dasgupta, T.; Saha, R.; Dey, L.; and Naskar, A. 2018. Automatic extraction of causal relations from text using linguistically informed deep neural networks. In SIGDIAL, 306-316. Devlin, J.; Chang, M.; Lee, K.; and Toutanova, K. 2018. BERT: pre-training of deep bidirectional transformers for language understanding. CoRR abs/1810.04805.

Do, Q.; Chan, Y. S.; and Roth, D. 2011. Minimally supervised event causality identification. In EMNLP, 294-303.

Halpern, J. Y. 2016. Actual Causality. The MIT Press.

Hassanzadeh, O.; Bhattacharjya, D.; Feblowitz, M.; Srinivas, K.; Perrone, M.; Sohrabi, S.; and Katz, M. 2019. Answering binary causal questions through large-scale text mining: An evaluation using cause-effect pairs from human experts. In IJCAI, 5003-5009.

Hassanzadeh, O.; Trewin, S.; and Gliozzo, A. 2018. Semantic concept discovery over event databases. In ESWC, 288-303.

Kruengkrai, C.; Torisawa, K.; Hashimoto, C.; Kloetzer, J.; Oh, J.-H.; and Tanaka, M. 2017. Improving event causality recognition with multiple background knowledge sources using multi-column convolutional neural networks. In AAAI, 3466-3473.

Mikolov, T.; Chen, K.; Corrado, G.; and Dean, J. 2013. Efficient estimation of word representations in vector space. CoRR abs/1301.3781.

Pearl, J. 2009. Causality: Models, Reasoning and Inference. New York, NY, USA: Cambridge University Press.

Radinsky, K.; Davidovich, S.; and Markovitch, S. 2012. Learning to predict from textual data. J. Artif. Intell. Res. 45:641-684.

Sharp, R.; Surdeanu, M.; Jansen, P.; Clark, P.; and Hammond, M. 2016. Creating causal embeddings for question answering with minimal supervision. In EMNLP, 138-148.

Sohrabi, S.; Katz, M.; Hassanzadeh, O.; Udrea, O.; Feblowitz, M. D.; and Riabov, A. 2019. IBM scenario planning advisor: Plan recognition as AI planning in practice. AI Commun. 32(1):1-13. 